Journal of Engineering and Applied Sciences 14 (Special Issue 7): 10180-10185, 2019

ISSN: 1816-949X

(c) Medwell Journals, 2019

\title{
Developed a Hydraulic Model as a Useful Tool in the Improvement of the Water Pipe Network in Al-Hilla City
}

\author{
Alaa Hussein Al-Fatlawi and Teeba Salih Merjan \\ College of Engineering, University of Babylon, Hillah, Iraq
}

\begin{abstract}
The main objective of this study is designing of Water Distribution Network (WDN) based on loops hydraulically. The existing water distribution network could not able to service the total area after the subsequent years due to population inflation and the increasing of water demand. Thus, new design of water distribution network suggested to insurance the population consumption and water demand of the present area after 25 years from base year of 2018. The data for the water pipe network such as the maps, diameters, material, utilities, land ordinates and the area supply method were obtained from Babylon Water Directorate.
\end{abstract}

Key words: Babylon, maps, population, hydraulically, utilities, ordinates

\section{INTRODUCTION}

The design of municipal Water supply and Distribution Systems (WDSs) is still practiced in professional studies using gross simplifying assumptions and a rigid-static analysis. As a result, the system is not tested under various conditions which can be encountered during its operational life and under different water demand scenarios. This may create frequent failures in meeting the actual demand or pressure requirements during the operation of the system operation in real life conditions. In the opposite direction the system is often over-designed and very costly. During the recent years the scientific community has produced a large number of innovative methods which can offer significant improvements in the design of WDSs. Apart from the improvements of well-known solvers for the analysis of WDSs (Jeppson, 1976; Larock et al., 2000; Tsakiris and Spiliotis, 2014), more realistic description of the conditions of nodal demands, roughness of pipes and their variability can also offer significantly in the accuracy of the results derived (Xu and Goulter, 1996; Spiliotis and Tsakiris, 2012 a). It is expected that even with the most conventional tools currently used in practice, some guidance for the assumptions and the range of values of some important parameters related to the materials used is still needed. In this context an attempt was recently made to produce and propose some practical guidelines for the analysis of WDSs, mainly targeted towards professional engineers (Tsakiris and Spiliotis, 2014). In short the five-generation classes and their basic characteristics are concisely presented in Table 1.
The recent developments for the analysis of WDSs (generation classes 3 and 4) incorporate (Tsakiris, 2014):

- The dependence of nodal outflow on the pressure at the node

- The uncertainty analysis of nodal outflow and pipe roughness

Major assumptions: In the professional practice the conventional design of an urban water supply and distribution system is based on a number of simplifying assumptions, the most critical of which are:

- The design discharge is based on the expected average demand of the estimated future population

- Empirical multipliers are used for estimating the maximum water demand

- The system is solved for steady state operation with the expected maximum demand

- The type of materials to be used is decided prior to the analysis of the system

- $\quad$ Pipe roughness and local energy losses are estimated using bibliographic data

- The internal diameters of the pipes are predetermined irrespective of the type of coating (e.g., internal coating in steel pipes has different depth if it is of epoxy resin or cement)

- Energy losses are calculated with the expected maximum water demands concentrated at the nodes, uniformly and simultaneously distributed

The major problem in this type of design is that all of the parameters involved are considered constant and 
Table 1: The five-generation classes methods for the analysis of WDSs Tsakiris (2014)

\begin{tabular}{lll}
\hline Generation class & Basic characteristics & Representative references \\
\hline 1 & Sequential solution of Q-equation or H-equations & Cross (1936) \\
& A linear system is solved in any iteration for & \\
& determining the $\Delta \mathrm{Q}$ and $\Delta \mathrm{h}$ & Wood and Charles (1972) \\
& Linear & Shamir and Howard (1968) \\
& Newton-Raphson & \\
& Pipe equations are solved for Q and h & Todini and Pilati (1988) \\
& Simultaneously (e.g., Gradient method/EPANET) & \\
& Analysis based on pressure dependent outflow \\
& (head driven simulation) & Tabesh et al. (2002) \\
& h-N-R Hazen-Williams & Giustolisi and Todini (2009) \\
& Linear flow along branches & Siew and Tanyimboh (2012) \\
& EPANET & Tsakiris and Spiliotis (2014) \\
& h-N-R Darcy-Weisbach & \\
& Taylor series expansion around the mean (for & Xu and Goulter (1996) \\
& Q or h) & Gupta and Bhare \\
& Fuzzy sets Q & Spiliotis and Tsakiris (2012)
\end{tabular}

reliable throughout the life cycle of the system. It is of outmost importance that at least few additional tests and procedures could be implemented for narrowing the range of systematic errors in the operation of these systems. These can be:

- The analysis of the entire system, water cycle analysis from the source to the end user

- The "dynamic" simulation of the system during a $24 \mathrm{~h}$ expected operation

- The testing of the system for scenarios representing demand concentration at certain parts of the system (e.g. where concentration of population is expected to increase or extension of the system seems likely to occur in the future)

- The description of the demand at the nodes and the pipe roughness by their range instead of constant values

Design criteria: Conventionally during the design of a WDS, the principal criterion is the fulfilment of flow and head requirements in all parts of the urban area. In most of the cases the water distribution system is isolated and analysed separately from the water supply network (from the source to the service reservoir).

However, even if the system incorporates the water supply and the distribution systems together, the criterion which is used is to find the solutions which lead to the minimum total construction costs. Based on this single criterion the source of water, the location of the service reservoirs and the dimensions of the mains are selected. Today in the new complicated world, obviously, this single criterion seems very weak if our objective is to rationally design the system for most reliable and effective operation during its whole life.

\section{MATERIALS AND METHODS}

Description of area: The area for which we are designing the water distribution system is Al-Hilla city. The population of the area is 692055 (Babylon census directorate, 2018). The distribution system designed, here is loop system. Water is supplied to the whole districts of Al-Soub Al-Kabeer via. a five separated pumping stations as shown in Fig. 1.

Estimation the population consumption: The data for the water-pipe network such as the maps, diameters, material, utilities, land ordinates and the area supply method were obtained from Babylon Water Directorate. Some suggestion were carried out in the design. Also, fire flow rate state in the design criteria shall be $0.95 \mathrm{~m}^{3} / \mathrm{min}\left(1368 \mathrm{~m}^{3} /\right.$ day) for each fire hydrant were calculated in Table 1 where the percentage of the total consumption of water Al-Hilla city was $450 \mathrm{~L}$ per capita per day. Table 2 show pump stations and the districts served and its demand.

EPANET2 program: EPANET2 is a computer program that performs extended period simulation of hydraulic and water quality behavior within pressurized pipe networks.

A network consists of pipes, nodes (pipe junctions), pumps, valves and storage tanks or reservoirs. EPANET2 tracks the flow of water in each pipe, the pressure at each node, the height of water in each tank and the concentration of a chemical species throughout the network during a simulation period comprised of multiple time steps. In addition, to chemical species, water age and source tracing can also be simulated. EPANET2 is 


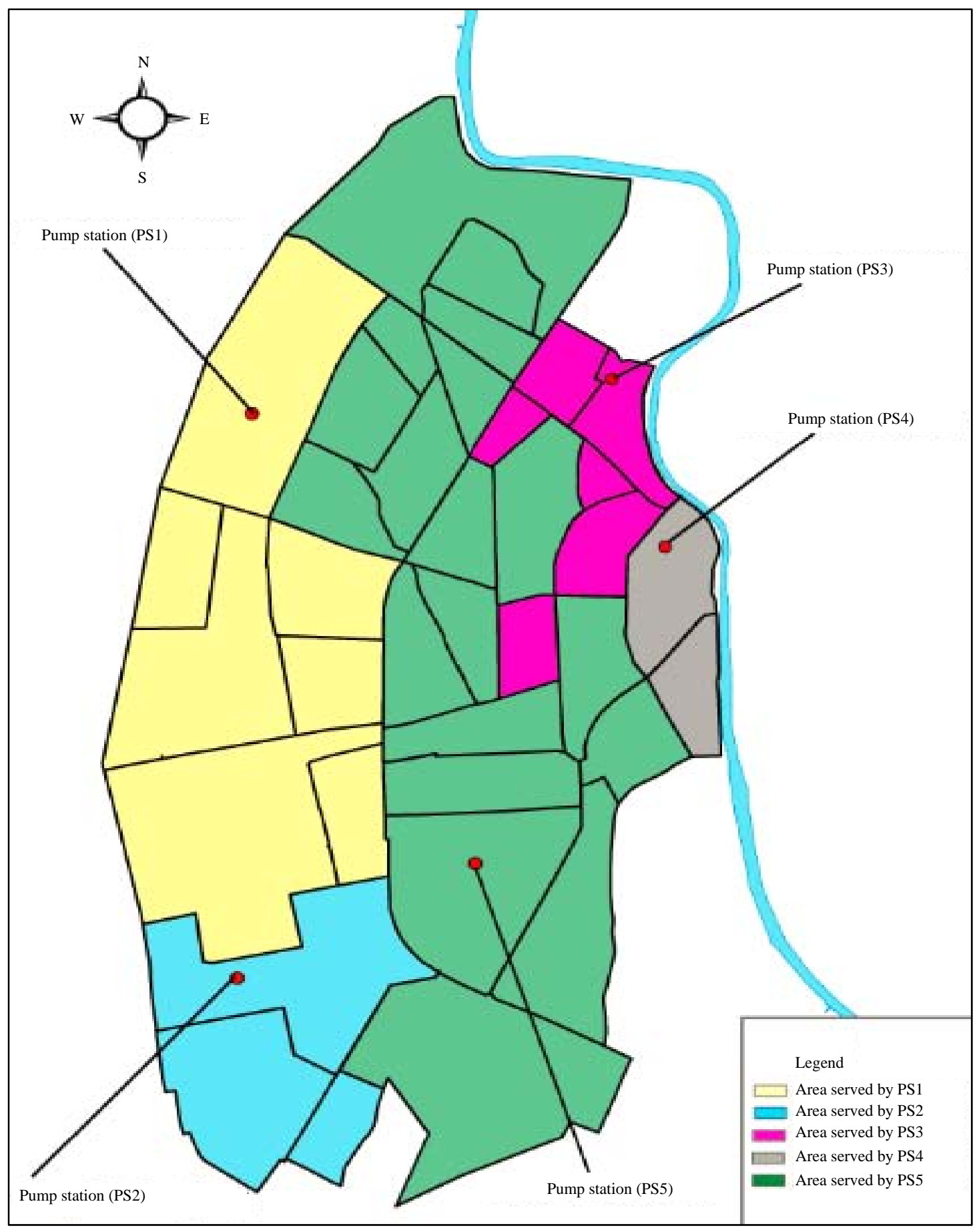

Fig. 1: Location of the pump stations and the area surved

designed to be a research tool for improving our understanding of the movement and fate of drinking water constituents within distribution systems. EPANET2 can help assess alternative management strategies for improving water quality throughout a system. These can include (Table 3):
- Altering source utilization within multiple source systems

- Altering pumping and tank filling/emptying schedules

- Use of satellite treatment such as re-chlorination at storage tanks

- Targeted pipe cleaning and replacement 
J. Eng. Applied Sci., 14 (Special Issue 7): 10180-10185, 2019

Table 2: Pump stations and the districts served and its demand

\begin{tabular}{|c|c|c|c|c|c|c|}
\hline $\begin{array}{l}\text { Pump } \\
\text { station }\end{array}$ & $\begin{array}{l}\text { Capacity } \\
\mathrm{m}^{3} / \mathrm{h}\end{array}$ & Districts & $\begin{array}{l}\text { Expected } \\
\text { pop., } 2043\end{array}$ & $\begin{array}{c}\text { Domestic demand } \\
\mathrm{m}^{3} / \mathrm{h}\end{array}$ & $\begin{array}{l}\text { Fire demand } \\
\mathrm{m}^{3} / \mathrm{h}\end{array}$ & $\begin{array}{l}\text { Total } \\
\mathrm{m}^{3} / \mathrm{h}\end{array}$ \\
\hline \multirow[t]{6}{*}{ Muhazim PS1 } & 2400 & Muhazim & 4907 & 92 & 57 & 149 \\
\hline & & 17 Nisan & 31601 & 592 & 57 & 649 \\
\hline & & Almealimin & 19483 & 365 & 57 & 422 \\
\hline & & Almuhandisn & 19356 & 362 & 57 & 419 \\
\hline & & Alaskari & 22435 & 420 & 114 & 534 \\
\hline & & Aleamarat Alsekeniah & 6365 & 119 & 57 & 176 \\
\hline \multirow{5}{*}{$\begin{array}{l}\text { Al-Tuhmazia } \\
\text { PS2 }\end{array}$} & 2400 & Al- Tuhmazia, Alakrmyn & 70888 & 1329 & & \\
\hline & & & & $51+1278$ & & 1 \\
\hline & & HamzaAldally & 10745 & (PS1+PS2) & 114 & $\begin{array}{r}1443 \\
258\end{array}$ \\
\hline & & Alsinaei & 20644 & 387 & 57 & 444 \\
\hline & & Naseege & 9156 & 171 & 57 & 228 \\
\hline \multicolumn{7}{|l|}{ Altiyaruh } \\
\hline \multirow[t]{7}{*}{ PS3 } & 1200 & Altiyaruh & 10292 & $\begin{array}{l}192 \\
78+114\end{array}$ & & \\
\hline & & & & (PS2+PS3) & 57 & 249 \\
\hline & & Alsaha & 6278 & 117 & 57 & 174 \\
\hline & & Altadamun & 7758 & 145 & 57 & 202 \\
\hline & & Albustan & 1192 & 22 & 57 & 79 \\
\hline & & Alkhadih & 12940 & 242 & 57 & 299 \\
\hline & & Aljameih & 6937 & 130 & 57 & 187 \\
\hline \multirow{3}{*}{ Aljabal PS4 } & 1200 & Alakrad, Almahdia & 33585 & 629 & & \\
\hline & & & & $88+541$ & & \\
\hline & & & & (PS3+PS4) & 57 & 686 \\
\hline Suggested water & & Aljamiein, Aljumhuri & 21996 & 412 & 57 & 469 \\
\hline \multirow[t]{21}{*}{ treatment plant PS5 } & 8000 & Mustafa ragib, Alibrahimia & 18733 & & & \\
\hline & & & & 351 & & \\
\hline & & & & $133+218$ & & \\
\hline & & & & (PS4+PS5) & 114 & 465 \\
\hline & & Alshawi & 28311 & 530 & 114 & 644 \\
\hline & & Alaskan & 20461 & 383 & 57 & 440 \\
\hline & & Nadir 3 & 16869 & 316 & 57 & 373 \\
\hline & & Nadir 1and Nadir 2, Alzahraa & 36622 & 686 & 57 & 743 \\
\hline & & Almurtada & 11238 & 210 & 57 & 267 \\
\hline & & Almukhabrat, Alsundubad & 11597 & 217 & 114 & 331 \\
\hline & & Alhussain & 11603 & 217 & 57 & 274 \\
\hline & & Faza'a and Manie & 34866 & 653 & 114 & 767 \\
\hline & & Alasatdhih & 19313 & 362 & 57 & 419 \\
\hline & & Alkaramih & 33440 & 627 & 114 & 741 \\
\hline & & Albuhtri & 5513 & 103 & 57 & 160 \\
\hline & & Alemam & 10483 & 196 & 57 & 253 \\
\hline & & Aldubat & 7636 & 143 & 57 & 200 \\
\hline & & Alshuhadaa & 14464 & 271 & 57 & 328 \\
\hline & & Almuharibin & 23800 & 446 & 57 & 503 \\
\hline & & Alsadr, Alaintifaduh & 52037 & 975 & 171 & 1146 \\
\hline & & Total & 692055 & 12613 & 2565 & 15178 \\
\hline
\end{tabular}

Table 3: Summary analysis of Al-Hilla city water network

\begin{tabular}{|c|c|c|c|c|c|c|c|c|}
\hline & & & & & & & Valves & \\
\hline Districts & Elevation (m) & Demand $\left(\mathrm{m}^{3} / \mathrm{h}\right)$ & Pipe type & Roughness & Diameter (mm) & Length (m) & ---------------------- & \\
\hline \multirow[t]{3}{*}{ Muhazim } & $27.30-28.62$ & 0.19 & DI & 120 & 200 & 38646 & Gate valve & 6 \\
\hline & & & PVC & 150 & 160 & 1228 & Air release valve & 3 \\
\hline & & & & & 100 & 38646 & Wash valve & 4 \\
\hline \multirow[t]{3}{*}{17 Nisan } & 27.11-27.70 & 0.84 & DI & 120 & 200 & 27640 & Gate valve & 6 \\
\hline & & & PVC & 150 & 200 & 1242 & Air release valve & 4 \\
\hline & & & & & 100 & 2981 & Wash valve & 3 \\
\hline \multirow[t]{3}{*}{ Albuhtri } & $27.11-27.70$ & 0.84 & DI & 120 & 200 & 3008 & Gate valve & 6 \\
\hline & & & PVC & 150 & 160 & 1096 & Air release valve & 3 \\
\hline & & & & & 100 & 25978 & Wash valve & 3 \\
\hline \multirow{4}{*}{$\begin{array}{l}\text { Aleamarat } \\
\text { Alsekeniah }\end{array}$} & & & & & & & & \\
\hline & $26-26.81$ & 1.89 & DI & 120 & 200 & 2898 & Gate valve & 6 \\
\hline & & & PVC & 150 & 160 & 1078 & Air release valve & 3 \\
\hline & & & & & 100 & 27842 & Wash valve & 3 \\
\hline Altuhmazia & $26.02-27.56$ & 1.09 & DI & 120 & 200 & 3278 & Gate valve & 6 \\
\hline Alakrmyn & & & PVC & 150 & 160 & 2780 & Air release valve & 5 \\
\hline
\end{tabular}


J. Eng. Applied Sci., 14 (Special Issue 7): 10180-10185, 2019

Table 3: Continue

\begin{tabular}{|c|c|c|c|c|c|c|c|c|}
\hline \multirow{3}{*}{ Districts } & \multirow[b]{2}{*}{ Elevation (m) } & \multirow[b]{2}{*}{ Demand $\left(\mathrm{m}^{3} / \mathrm{h}\right)$} & \multirow{3}{*}{ Pipe type } & \multirow[b]{2}{*}{ Roughness } & \multirow[b]{2}{*}{ Diameter (mm) } & \multirow{3}{*}{ Length (m) } & \multicolumn{2}{|l|}{ Valves } \\
\hline & & & & & & & & \\
\hline & & & & 100 & & & Wash valve & 4 \\
\hline \multirow[t]{3}{*}{ Alaskan } & 27.01- 27.99 & 0.64 & DI & 120 & 200 & 1897 & Gate valve & 4 \\
\hline & & & PVC & 150 & 160 & 765 & Air release valve & 3 \\
\hline & & & & 100 & & 27842 & Wash valve & 3 \\
\hline \multirow[t]{3}{*}{ Alshawi } & $27.01-27.67$ & 1.03 & DI & 120 & 200 & 2898 & Gate valve & 6 \\
\hline & & & PVC & 150 & 160 & 1101 & Air release valve & 3 \\
\hline & & & & 100 & & 43009 & Wash valve & 4 \\
\hline Almurtada & $27-27.82$ & 0.31 & DI & 120 & 200 & 2432 & Gate valve & 5 \\
\hline & & & PVC & 150 & 160 & 972 & Air release valve & 3 \\
\hline & & & & 100 & & 63250 & Wash valve & 3 \\
\hline Aljameih & $27-27.92$ & 0.15 & DI & 120 & 200 & 4008 & Gate valve & 5 \\
\hline & & & PVC & 150 & 160 & 1885 & Air release valve & 3 \\
\hline & & & & 100 & & 29321 & Wash valve & 5 \\
\hline Almealimin & $26.10-26.76$ & 0.45 & DI & 120 & 200 & 2378 & Gate valve & 4 \\
\hline & & & PVC & 150 & 160 & 1006 & Air release valve & 4 \\
\hline & & & & 100 & & 9549 & Wash valve & 3 \\
\hline Alasatdhih & 26.09-26.99 & 1.12 & DI & 120 & 200 & 2778 & Gate valve & 4 \\
\hline & & & PVC & 150 & 160 & 1028 & Air release valve & 3 \\
\hline & & & & 100 & & 54776 & Wash valve & 3 \\
\hline Hamza & $27-27.88$ & 0.77 & DI & 120 & 200 & 3006 & Gate valve & 5 \\
\hline Aldally & & & PVC & 150 & 160 & 1897 & Air release valve & 3 \\
\hline & & & & 100 & & 6890 & Wash valve & 3 \\
\hline Almuhandisn & $26.01-26.83$ & 0.97 & DI & 120 & 200 & 31452 & Gate valve & 4 \\
\hline & & & PVC & 150 & 160 & 1087 & Air release valve & 4 \\
\hline & & & & 100 & & 31452 & Wash valve & 3 \\
\hline Alaskari & 27.07-27.95 & 0.34 & DI & 120 & 200 & 3094 & Gate valve & 6 \\
\hline & & & PVC & 150 & 160 & 1888 & Air release valve & 4 \\
\hline & & & & 100 & & 30892 & Wash valve & 4 \\
\hline Faza'a and & 27.17-27.85 & 0.45 & DI & 120 & 200 & 1569 & Gate valve & 5 \\
\hline Manie & & & PVC & 150 & 160 & 799 & Air release valve & 3 \\
\hline & & & & 100 & & 19876 & Wash valve & 3 \\
\hline Nadir 3 & $27.09-27.82$ & 0.69 & DI & 120 & 200 & 2844 & Gate valve & 4 \\
\hline & & & PVC & 150 & 160 & 1033 & Air release valve & 3 \\
\hline & & & & 100 & & 29041 & Wash valve & 3 \\
\hline Nadir1, Nadir & 27.04-27.89 & 1.35 & DI & 120 & 200 & 1476 & Gate valve & 6 \\
\hline 2, Alzahraa & & & PVC & 150 & 160 & 543 & Air release valve & 3 \\
\hline & & & & 100 & & 22342 & Wash valve & 3 \\
\hline Alsinaei & 27.14-27.69 & 0.64 & DI & 120 & 200 & 1432 & Gate valve & 4 \\
\hline & & & PVC & 150 & 160 & 508 & Air release valve & 2 \\
\hline & & & & & 100 & 13862 & Wash valve & 2 \\
\hline Naseege & 26.01-26.99 & 0.15 & DI & 120 & 200 & 6789 & Gate valve & 9 \\
\hline & & & PVC & 150 & 160 & 2701 & Air release valve & 3 \\
\hline & & & & & 100 & 49780 & Wash valve & 5 \\
\hline Alsundubad & 27.13-27.70 & 0.19 & DI & 120 & 200 & 1084 & Gate valve & 7 \\
\hline Almukhabrat & & & PVC & 150 & 160 & 2351 & Air release valve & 3 \\
\hline & & & & & 100 & 33908 & Wash valve & 4 \\
\hline Alkadhia & $26.54-27.60$ & 0.05 & DI & 120 & 200 & 2908 & Gate valve & 6 \\
\hline & & & PVC & 150 & 160 & 1089 & Air release valve & 3 \\
\hline & & & & & 100 & 68984 & Wash valve & 3 \\
\hline Altiyaruh & 27.28-27.99 & 0.11 & DI & 120 & 200 & 3412 & Gate valve & 6 \\
\hline & & & PVC & 150 & 160 & 2365 & Air release valve & 3 \\
\hline & & & & & 100 & 26800 & Wash valve & 3 \\
\hline Alsaha & $27.10-27.44$ & 0.21 & DI & 120 & 200 & 3003 & Gate valve & 4 \\
\hline & & & PVC & 150 & 160 & 1144 & Air release valve & 3 \\
\hline & & & & & 100 & 6947 & Wash valve & 3 \\
\hline Alsadr & $27.3-28$ & & & & & & & \\
\hline Alaintifaduh & & 0.47 & DI & 120 & 200 & 6090 & Gate valve & 12 \\
\hline & & & PVC & 150 & 160 & 3365 & Air release valve & 6 \\
\hline & & & & & 100 & 79826 & Wash valve & 7 \\
\hline Alemam & $27-27.77$ & 0.18 & DI & 120 & 200 & 2765 & Gate valve & 6 \\
\hline & & & PVC & 150 & 160 & 1055 & Air release valve & 4 \\
\hline & & & & & 100 & 29992 & Wash valve & 5 \\
\hline Aldubat & $27.12-27.66$ & 0.50 & DI & 120 & 200 & 2654 & Gate valve & 5 \\
\hline & & & PVC & 150 & 160 & 985 & Air release valve & 4 \\
\hline & & & & & 100 & 23091 & Wash valve & 3 \\
\hline Alshuhada & $27-27.94$ & 0.34 & DI & 120 & 200 & 2654 & Gate valve & 6 \\
\hline & & & PVC & 150 & 160 & 985 & Air release valve & 5 \\
\hline & & & & & 100 & 32091 & Wash valve & 5 \\
\hline
\end{tabular}


Table 3: Continue

\begin{tabular}{|c|c|c|c|c|c|c|c|c|}
\hline & & & & & & & Valves & \\
\hline Districts & Elevation (m) & Demand $\left(\mathrm{m}^{3} / \mathrm{h}\right)$ & Pipe type & Roughness & Diameter (mm) & Length (m) & -------------- & \\
\hline \multirow[t]{3}{*}{ Almuharibin } & $26-27.16$ & 0.53 & DI & 120 & 200 & 3008 & Gate valve & 7 \\
\hline & & & PVC & 150 & 160 & 1096 & Air release valve & 6 \\
\hline & & & & & 100 & 28978 & Wash valve & 5 \\
\hline Alakrad & $27-27.87$ & 1.15 & DI & 120 & 200 & 2801 & Gate valve & 5 \\
\hline \multirow[t]{2}{*}{ Almahdia } & & & PVC & 150 & 160 & 987 & Air release valve & 4 \\
\hline & & & & & 100 & 29242 & Wash valve & 3 \\
\hline Aljamiein & $26-26.98$ & 1.95 & DI & 120 & 200 & 2908 & Gate valve & 4 \\
\hline \multirow[t]{2}{*}{ Aljumhuriu } & & & PVC & 150 & 160 & 1090 & Air release valve & 3 \\
\hline & & & & & 100 & 27880 & Wash valve & 3 \\
\hline Mustafa ragib & $26.10-26.97$ & 1.19 & DI & 120 & 200 & 3610 & Gate valve & 4 \\
\hline \multirow[t]{2}{*}{ Alibrahimia } & & & PVC & 150 & 160 & 2205 & Air release valve & 3 \\
\hline & & & & & 100 & 36128 & Wash valve & 3 \\
\hline \multirow[t]{3}{*}{ Alhussain } & 27-27.99 & 0.25 & DI & 120 & 200 & 2955 & Gate valve & 6 \\
\hline & & & PVC & 150 & 160 & 1160 & Air release valve & 3 \\
\hline & & & & & 100 & 29077 & Wash valve & 3 \\
\hline \multirow[t]{3}{*}{ Alkaramih } & 26.10-26.92 & 0.97 & DI & 120 & 200 & 1657 & Gate valve & 7 \\
\hline & & & PVC & 150 & 160 & 890 & Air release valve & 4 \\
\hline & & & & & 100 & 45204 & Wash valve & 5 \\
\hline \multirow[t]{3}{*}{ Albusatan } & $27-27.82$ & 0.04 & DI & 120 & 200 & 1011 & Gate valve & 4 \\
\hline & & & PVC & 150 & 160 & 3821 & Air release valve & 3 \\
\hline & & & & & 100 & 36036 & Wash valve & 2 \\
\hline \multirow[t]{3}{*}{ Altadamun } & 27-27.91 & 0.15 & DI & 120 & 200 & 2801 & Gate valve & 4 \\
\hline & & & PVC & 150 & 160 & 1050 & Air release valve & 3 \\
\hline & & & & & 100 & 26687 & Wash valve & 3 \\
\hline
\end{tabular}

\section{RESULTS AND DISCUSSION}

Before building a model, it is necessary to gather information describing the network. Introduces and discusses sources of data used in constructing the models, also presented the simulation of the EPAENT2 Model for the case study area. Table 3 shows summary analysis of area under study water network.

\section{CONCLUSION}

In this research, the water distribution system has been design with the help of EPANET2 in which we use number of nodes, number of pipe, elevation, demands, diameter and length of Al-Hilla city. Once successful run is done using EPANET2 tool after simulation. Design of the water supply scheme for proper supply of water is efficient to meet the daily requirement of water. The selected pipe sizes for suggested water distribution network is adequate and will meet avarage water demands and fire protection while maintaining adequate or prescribed pressure in the system.

\section{REFERENCES}

Cross, H., 1936. Analysis of Flow in Networks of Conduits or Conductors. University of Illinois at Urbana-Champaign, Illinois,.

Giustolisi, O. and E. Todini, 2009. Pipe hydraulic resistance correction in WDN analysis. Urban Water J., 6: 39-52.

Jeppson, R.W., 1976. Analysis of Flow in Pipe Networks. Ann Arbor Science Publishers, Inc., Collingwood.
Larock, B., R. Jeppson and G. Watters, 2000. Hydraulics of Pipeline Systems. CRS Press, Boca Raton, Florida, USA.,.

Shamir, U. and C.D. Howard, 1968. Water distribution system analysis. J. Hydraul. Eng., 94: 219-234.

Siew, C. and T.T. Tanyimboh, 2012. Pressure-dependent EPANET extension. Water Resour. Manage., 26: $1477-1498$.

Spiliotis, M. and G. Tsakiris, 2012. Water distribution network analysis under fuzzy demands. Civil Eng. Environ. Syst., 29: 107-122.

Tabesh, M., T.T. Tanyimboh and R. Burrows, 2002. Head-driven simulation of water supply networks. Intl. J. Eng. Trans. A. Basics, 15: 11-22.

Todini, E. and S. Pilati, 1988. A Gradient Algorithm for the Analysis of Pipe Networks. In: Computer Applications in Water Supply, Coulbeck, B. and O. Chun-Hou (Eds.). Wiley, Hoboken, New Jersey, USA., pp: 1-20.

Tsakiris, G. and M. Spiliotis, 2014. A newton-raphson analysis of urban water systems based on nodal head-driven outflow. Eur. J. Environ. Civil Eng., 18: 882-896.

Tsakiris, G., 2014. Rational design of urban water supply and distribution systems. Water Util. J., 8: 5-16

Wood, D.J. and C.O. Charles, 1972. Hydraulic network analysis using linear theory. J. Hydraul. Eng., 98: $1157-1170$.

Xu, C. and I.C. Goulter, 1996. Uncertainty Analysis of Water Distribution Networks. In: Stochastic Hydraulics 96, Tickle, K.S., I.C. Goulter, C. Xu, S.A. Wasimi and F. Bouchart (Eds.). August Aime Balkema, Amsterdam, South Africa, ISBN:9789054108177, pp: 609-616. 